\title{
Limits on the isotropic diffuse $\gamma$-rays at ultra high energies measured with KASCADE
}

\author{
Z. Feng ${ }^{* 1}$, W.D. Apel ${ }^{2}$, J.C. Arteaga-Velázquez ${ }^{3}$, K. Bekk ${ }^{2}$, M. Bertaina ${ }^{4}$, J. Blümer ${ }^{2,5}$, \\ H. Bozdog ${ }^{2}$, I.M. Brancus ${ }^{6}$, E. Cantoni ${ }^{4,7}$, A. Chiavassa $^{4}$, F. Cossavella ${ }^{5}$, \\ K. Daumiller ${ }^{2}$, V. de Souza ${ }^{8}$, F. Di Pierro ${ }^{4}$, P. Doll ${ }^{2}$, R. Engel ${ }^{2}$, D. Fuhrmann ${ }^{9}$, \\ A. Gherghel-Lascu ${ }^{6}$, H.J. Gils ${ }^{2}$, R. Glasstetter ${ }^{9}$, C. Grupen ${ }^{10}$, A. Haungs ${ }^{2}$, D. Heck ${ }^{2}$, \\ J.R. Hörandel ${ }^{11}$, D. Huber ${ }^{5}$, T. Huege ${ }^{2}$, K.-H. Kampert ${ }^{9}$, D. Kang ${ }^{5}$, H.O. Klages ${ }^{2}$, \\ K. Link ${ }^{5}$, P. Łuczak ${ }^{12}$, H.J. Mathes ${ }^{2}$, H.J. Mayer ${ }^{2}$, J. Milke ${ }^{2}$, B. Mitrica ${ }^{6}$, C. Morello ${ }^{7}$, \\ J. Oehlschläger ${ }^{2}$, S. Ostapchenko ${ }^{13}$, N. Palmieri ${ }^{5}$, T. Pierog ${ }^{2}$, H. Rebel ${ }^{2}$, M. Roth ${ }^{2}$, \\ H. Schieler ${ }^{2}$, S. Schoo ${ }^{2}$, F.G. Schröder ${ }^{2}$, O. Sima ${ }^{14}$, G. Toma ${ }^{6}$, G.C. Trinchero ${ }^{7}$, \\ H. Ulrich ${ }^{2}$, A. Weindl ${ }^{2}$, J. Wochele ${ }^{2}$, J. Zabierowski ${ }^{12}$ - KASCADE-Grande \\ Collaboration \\ ${ }^{1}$ Institute of High Energy Physics, Beijing, China \\ ${ }^{2}$ Institut für Kernphysik, KIT - Karlsruhe Institute of Technology, Germany \\ ${ }^{3}$ Universidad Michoacana, Inst. Física y Matemáticas, Morelia, Mexico \\ ${ }^{4}$ Dipartimento di Fisica, Università degli Studi di Torino, Italy \\ ${ }^{5}$ Institut für Experimentelle Kernphysik, KIT - Karlsruhe Institute of Technology, Germany \\ ${ }^{6}$ Horia Hulubei National Institute of Physics and Nuclear Engineering, Bucharest, Romania \\ ${ }^{7}$ Osservatorio Astrofisico di Torino, INAF Torino, Italy \\ ${ }^{8}$ Universidade São Paulo, Instituto de Física de São Carlos, Brasil \\ ${ }^{9}$ Fachbereich Physik, Universität Wuppertal, Germany \\ ${ }^{10}$ Department of Physics, Siegen University, Germany \\ ${ }^{11}$ Dept. of Astrophysics, Radboud University Nijmegen, The Netherlands \\ ${ }^{12}$ National Centre for Nuclear Research, Department of Astrophysics, Lodz, Poland \\ ${ }^{13}$ Frankfurt Institute for Advanced Studies (FIAS), Frankfurt am Main, Germany \\ ${ }^{14}$ Department of Physics, University of Bucharest, Bucharest, Romania
}

\section{E-mail: fengzy@ihep.ac.cn, donghwa.kangekit.edu}

Using data measured by the KASCADE air shower array between 1996 October and 2010 May, an $90 \%$ C.L. upper limit to the flux of ultrahigh energy $\gamma$-rays in the primary cosmic ray flux is determined, at energies between $\sim 2.5 \times 10^{14} \mathrm{eV}$ and $\sim 1.9 \times 10^{16} \mathrm{eV}$. The upper limit of the fraction of $\gamma$-rays at $1.5 \times 10^{15} \mathrm{eV}$ is $1.7 \times 10^{-5}$, while $1.1 \times 10^{-5}$ at $3.7 \times 10^{15} \mathrm{eV}$. These are the lowest upper limits in the world up to now, which may set some constraints on the fundamental astrophysical model, such as the distance of sources for the IceCube excess model.

The 34th International Cosmic Ray Conference,

30 July- 6 August, 2015

The Hague, The Netherlands 


\section{Introduction}

$\gamma$-rays represent a very small but also very important fraction of primary cosmic rays. Their importance derives from the fact that they, other than the charged particles which constitute the bulk of cosmic ray primaries, are not deflected by interstellar magnetic fields. Hence their direction of incidence on the Earth points back to their origin.

Diffuse $\gamma$-rays are the sum of contributions from several components. One is the cascading products by the collision of cosmic rays with interstellar gas and dust in the disk of the Galaxy [四]. In this case, the predicted integral intensity is concentrated in the galactic plane. The other one is electromagnetic cascades induced by the interaction of ultra high energy cosmic rays (UHECRs) with the cosmic microwave background radiation [[] and the unresolved point sources in extra galactic astronomical objects [B], In this case, this results in an isotropic flux of secondary photons uniformly.

Duo to the low flux, direct detection of ultrahigh $\gamma$-rays is difficult. Fortunately, the detection can be accomplished by large area ground-based Extensive Air Shower (EAS) arrays. This paper reports a sensitive search for diffuse gamma rays and the upper limits of the isotropic flux based on the observations with the KASCADE.

\section{The KASCADE experiment and data selection}

The KArlsruhe Shower Core and Array DEtector (KASCADE) was located at Karlsruhe Institute of Technology, Germany $\left(8.4^{\circ} \mathrm{E}, 49.1^{\circ} \mathrm{N}\right)$ at $110 \mathrm{~m}$ above sea level, corresponding to an average vertical atmospheric depth of $1022 \mathrm{~g} / \mathrm{cm}^{2}$. The experiment measures the electromagnetic, muonic, and hadronic components of extensive air showers with three major detector systems: a large field array, a muon tracking detector, and a central detector. A detailed description of the KASCADE experiment can be found in [四].

In this analysis, data from the $200 \mathrm{~m} \times 200 \mathrm{~m}$ scintillation detector array are used. The 252 detector stations are uniformly spaced on a square grid of $13 \mathrm{~m}$ divisions. The stations are organized in 16 electronically independent socalled clusters, with 16 stations in the 12 outer clusters and 15 stations in the 4 inner clusters. The stations in the inner and outer clusters contain four and two liquid scintillator detectors, respectively, covering a total area of $490 \mathrm{~m}^{2}$. In addition, plastic scintillators are mounted below an absorber of $10 \mathrm{~cm}$ of lead and $4 \mathrm{~cm}$ of iron in the 192 stations of the outer clusters ( $622 \mathrm{~m}^{2}$ total area). The absorber corresponds to 20 electromagnetic radiation lengths, entailing a threshold for vertical muons of $230 \mathrm{MeV}$. This configuration allows the measurement of the electromagnetic and muonic components of extensive air showers. The angular resolution of KASCADE is better than $0.55^{\circ}$ for electron numbers $\log _{10} N_{e}>4$. . The trigger rate is about $3 \mathrm{~Hz}$.

Applying an iterative shower reconstruction procedure, the numbers of electrons and muons in a shower are determined basically by maximizing a likelihood function describing the measure-

\footnotetext{
* Speaker.

${ }^{\dagger}$ Z. Feng Sincerely thank KASCADE collaboration for allowing him to use all the data of KASCADE experiment. Z. Feng is supported by the Natural Sciences Foundation of China (Nos. 11405182), and by the Chinese Academy of Sciences (524014IHEPZZBS301).
} 
ments with the Nishimura-Kamata-Greisen (NKG) formula [ [ $]$ [ [6], assuming a Moliere radius of $89 \mathrm{~m}$. Detector signals are corrected for contributions of other particles, i.e., the electromagnetic detectors for contributions of muons, $\gamma$-rays, and hadrons [ [ $]$ ]. Shower directions and, hence, the directions of the incoming primary particles are determined without assuming a fixed geometrical shape of the shower front by evaluating the arrival times of the first particle in each detector and the total particle number per station.

In this analysis, in order to clean the data set of poorly reconstructed showers and obtain the precise muon number measurement, the following cuts were applied: all the 16 clusters must be working, shower core positions have to lay inside a circular area of $91 \mathrm{~m}$ radius around the center of the array to omit large reconstruction errors at the edges of the detector field, and zenith angles are requested to be $\theta<20^{\circ}$. The data set was recorded between 1996 October and 2010 May, corresponding to an effective time of about 4223.6 days. About $1.0 \times 10^{8}$ events are left for the analysis after the mentioned cuts.

\section{Monte Carlo study and gamma hadron discrimination}

To reproduce the observed muon number distribution and to estimate the median $\gamma$-ray energy , a full Monte Carlo (MC) simulation has been carried out on the development of air showers in the atmosphere by CORSIKA [8] with QGSjet-II-2 and Fluka interaction models. To determine the signals in the individual detectors, all secondary particles at the ground level are passed through a complete detector simulation program using the GEANT package. Showers initiated by primary $\gamma$-rays have been simulated. The $\gamma$-rays air shower events are generated with zenith angle less than $60^{\circ}$ with a differential energy spectrum $E^{-2.7}$ below $4 \times 10^{15} \mathrm{eV}$ (knee) and $E^{-3}$ above knee, assuming the knee is located at $4 \times 10^{15} \mathrm{eV}$ [9]. Energy of $\gamma$-rays is from $3 \times 10^{14} \mathrm{eV}$ to $6 \times 10^{16}$ $\mathrm{eV}$.

To study the trigger efficiency and the uncertainties of the energy estimation, CRs-induced showers initiated by primary protons, $\mathrm{He}, \mathrm{CNO}, \mathrm{Si}$ and iron nuclei have been simulated. cosmic rays air shower events are generated with zenith angles from $0^{\circ}$ to $50^{\circ}$, and azimuth from $0^{\circ}$ to $360^{\circ}$, where the energy range is from $10^{14} \mathrm{eV}$ to $10^{18} \mathrm{eV}$.

The energy of CRs is estimated from the experimental data. The formulas based on the measurement of the muon and electron numbers of the KASCADE array [ए]] is used. The uncertainties of this energy reconstruction formulas have been studied with Monte Carlo simulations. For events of this selection, the typical uncertainty is about $\sim 30 \%$ for individual events, which depends on the composition.

The trigger and reconstruction effciency of KASCADE as a function of the shower size, i.e. the number of electrons, is demonstrated in Figure $\mathbb{W}$. The detector array reaches full efficiency on the detection of showers for electron numbers $\log _{10} N_{e}>4$ for air showers induced by $\gamma$-rays, protons and iron primary particles, which approximately corresponds to a primary energy of $2.5 \times 10^{14} \mathrm{eV}$ for $\gamma$-rays and $3.3 \times 10^{14} \mathrm{eV}$ for CRs.

Figure $\square$ shows the distribution of electron versus truncated muon number $\left(\log _{10} N_{\mu, t r}-\log _{10} N_{e}\right)$ for measured showers and MC $\gamma$-ray-induced showers. The blue data points indicate the whole experimental data set, whereas the red one illustrates the simulated $\gamma$-ray events. Here $N_{\mu, t r}$ denotes the number of muons in the distance range of 40-200 $\mathrm{m}$ from the shower core. The electron number 


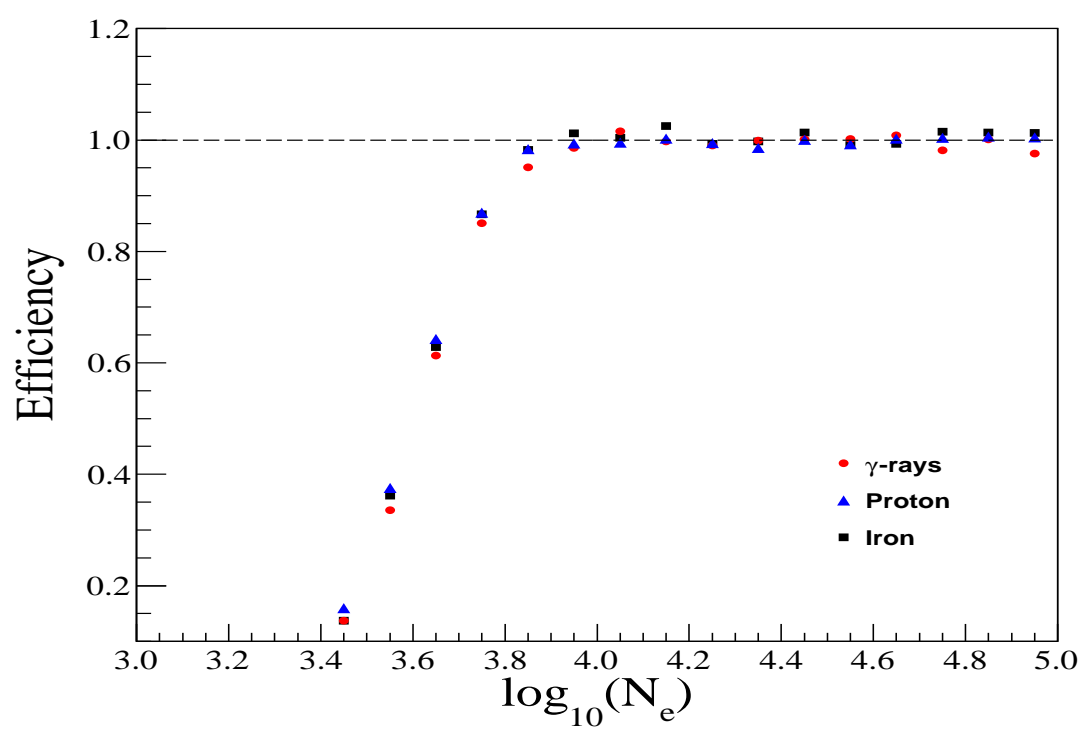

Figure 1: Trigger and reconstruction efficiency as a function of the number of electrons for air showers induced by photons, protons and iron primaries.

$N_{e}$ is corrected to a zenith angle of $12.7^{\circ}$ using an attenuation length of $\Lambda_{N_{e}}=158 \mathrm{~g} / \mathrm{cm}^{2}$ [W]]. In Figure 凤, the muonless showers are plotted at the position with $\log _{10} N_{\mu, t r}=1.5$.

The distribution of shower sizes for $\gamma$-ray-induced showers motivates the following cuts to select the muon-poor showers: $\log _{10} N_{\mu, t r}<2$ for $\log _{10} N_{e}<5.15$, and $\log _{10} N_{\mu, t r}<1.4 * \log _{10} N_{e}-$ 5.21 for $\log _{10} N_{e}>5.15$. This selection of the muon-poor showers is indicated by straight lines in Figure [].

\section{The analysis and results}

There is no possible excess of events consistent with a gamma-ray signal seen in the data. Hence, we assume that all events below the selection line are primary $\gamma$-rays and set upper limits on the gamma-ray fraction of the cosmic rays, which is a very conservative way in which the expected background is not subtracted from the event number below the cut line.

We estimate $N_{90}$, the $90 \%$ C.L. upper limit on the number of detected events, using standard statistical methods [ए2] and use the simulation to evaluate the efficiency for $\gamma$-ray detection, $\varepsilon_{\gamma}$, when the muon cut is applied. The upper limit on the fraction of the $\gamma$-ray integral flux relative to the cosmic ray integral flux, $I_{\gamma} / I_{C R}$, is given by [एँ3]

$$
\frac{I_{\gamma}}{I_{C R}}<\frac{N_{90}}{N_{t o t} \varepsilon_{\gamma}}\left(\frac{E_{C R}}{E_{\gamma}}\right)^{-\beta+1}
$$

where $E_{C R}$ is the mean cosmic ray energy, $E_{\gamma}$ is the mean gamma-ray energy, and $\beta$ is the integral cosmic-ray spectral index $\left(\beta=-2.7, E_{\gamma}<4 \times 10^{16} \mathrm{eV} ; \beta=3.0, E_{\gamma}>4 \times 10^{16} \mathrm{eV}\right)$. The mean energies of cosmic-rays and gamma-rays are calculated bin by bin using the resulting log-gaussian function. The factor involving energies in this equation accounts for the fact that $\gamma$-ray primaries produce larger showers than cosmic-ray primaries of the same energy, or conversely, that $\gamma$-ray showers are 


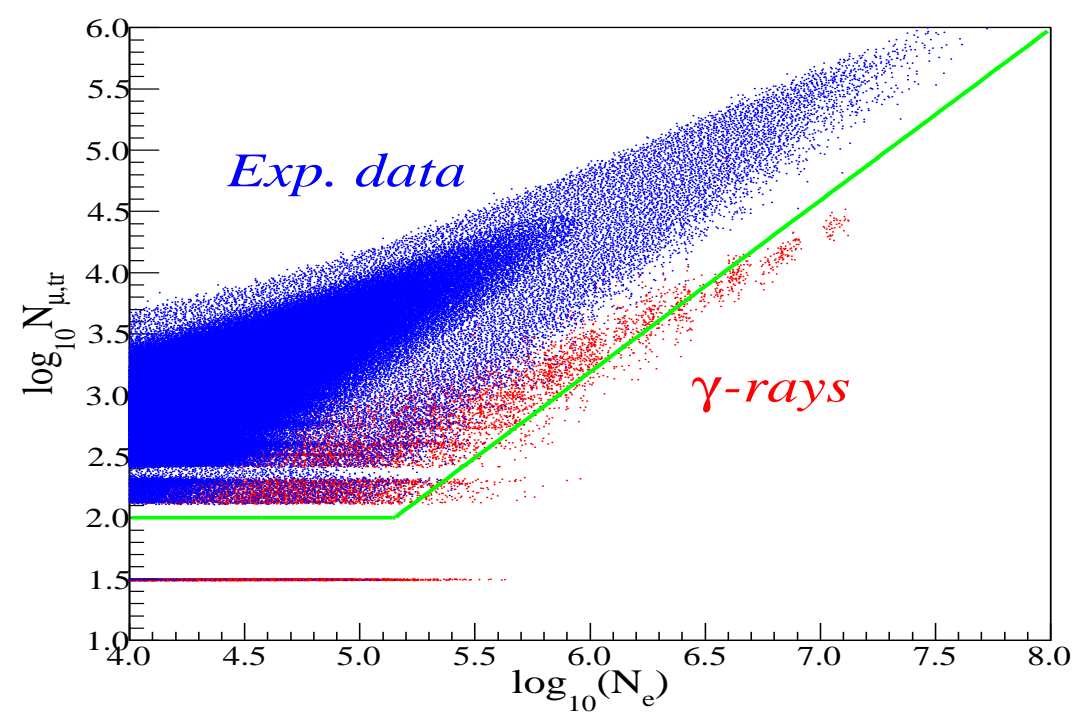

Figure 2: Scatter distribution of the measured number of muons $\log _{10} N_{\mu}$ and number of electrons $\log _{10} N_{e}$ superimposed with simulated gamma-ray showers. The green line indicates the choice of the cut for the selection of the muon-poor showers.

detected with the same efficiency as cosmic ray showers of higher energy. Therefore, to report a flux ratio at a fixed cosmic ray energy, we correct the $\gamma$-ray flux at its energy to the higher cosmicray energy under the assumption that both species have the same spectral index.

Table $\mathbb{W}$ lists the the values of $N_{\text {tot }}, N_{90}, \varepsilon_{\gamma}, E_{C R}, E_{\gamma}$ and $I_{\gamma} / I_{C R}$ of different threshold values of $\log _{10} N_{e}$. To determine upper limits on the diffuse $\gamma$-ray flux at fixed $\gamma$-ray energies, measurements

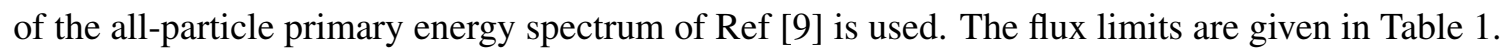
Figure [ $]$ shows a compilation of measurements on the $\gamma$-ray fraction as a function of energy, including this work, from $10 \mathrm{TeV}$ to $1 \mathrm{EeV}$. The upper limit of the fraction of $\gamma$-rays at $1.5 \times 10^{15} \mathrm{eV}$ is $1.7 \times 10^{-5}$, while $1.1 \times 10^{-5}$ at $3.7 \times 10^{15} \mathrm{eV}$. These are the lowest upper limits in the world up to now.

It should be noted that all values in Figure B] are limits, except that one from MSU [20]. In reference[ [D]], based on full data sets measured by KASCADE-Grands, the upper limit of the fraction of $\gamma$-rays is extended to $1 \mathrm{EeV}$.

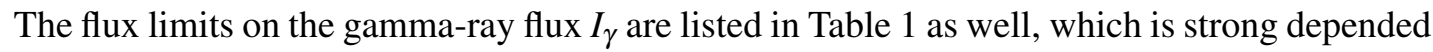
on the all-particle primary energy spectrum by each experiments. A comparison of the limits on the integral gamma-ray flux $I_{\gamma}$ with other previous experiments[22] is under investigation. Figures 9 shows the comparison of integral flux of $\gamma$-rays withe previous results and with theoretical curves by an IceCube neutrino excess model[[23] .The lines are IceCube excess model coming from different distances of sources of neutrinos in the galaxy, where these neutrinos are also responsible for primary gamma-rays. The flux limits on the gamma-ray flux $I_{\gamma}$ of this work at $1.5 \times 10^{15} \mathrm{eV}$ and $3.7 \times 10^{15} \mathrm{eV}$ lower than the theoretical prediction of the IceCube excess model coming from 20 kpc. This result strongly set some constrains on the distance of sources for the IceCube neutrino excess model [[23]]. 


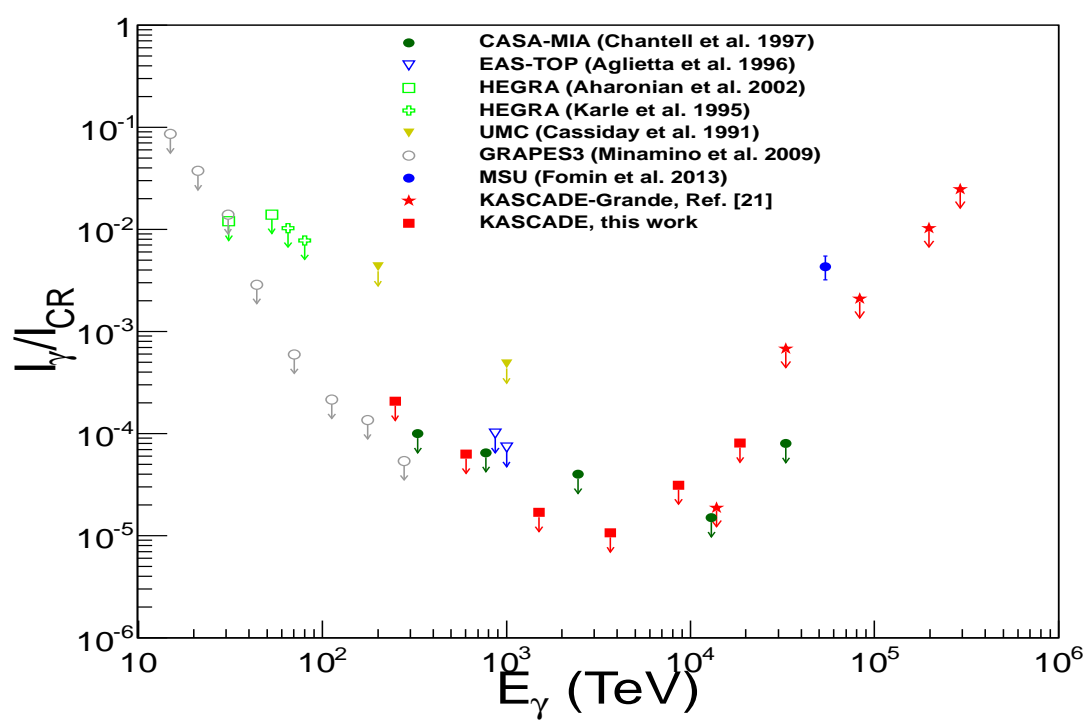

Figure 3: Measurements of the fraction of $\gamma$-rays relative to cosmic rays at ultrahigh energies. The points

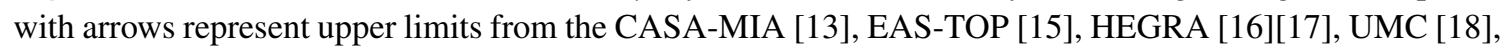

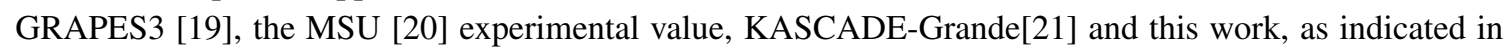
the legend.

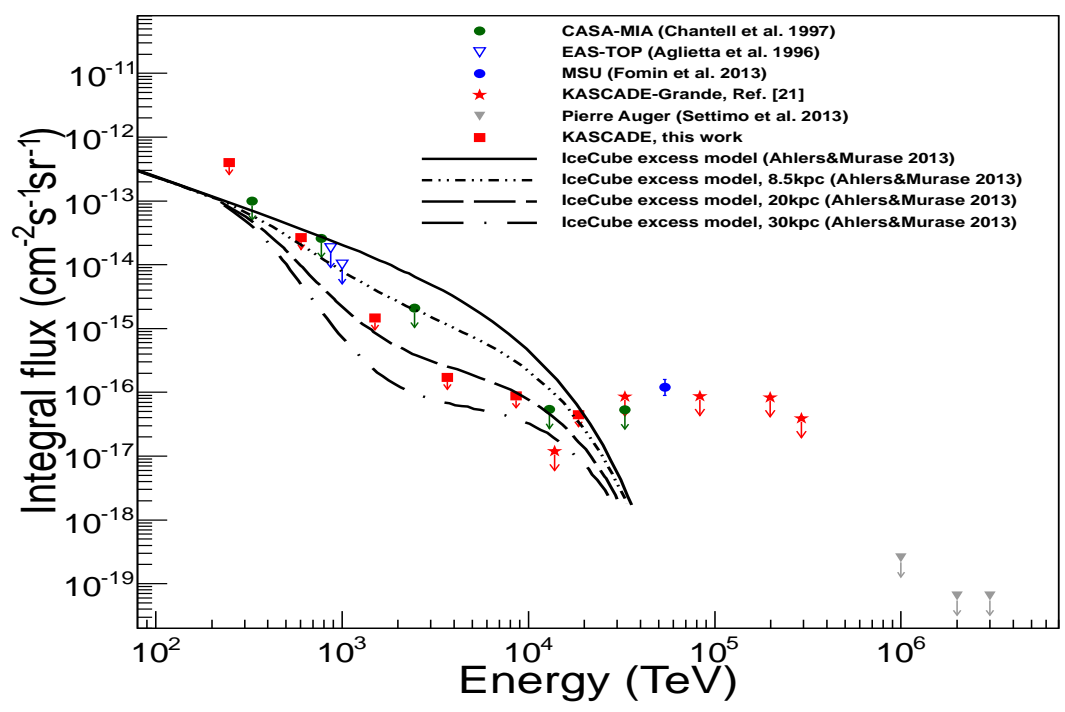

Figure 4: Comparison of integral flux of $\gamma$-rays with previous results and with theoretical vurves by an IceCube neutrino excess model[23] 
Table 1: Results of the search for diffuse ultra-high energy $\gamma$-rays at different threshold values of $\log _{10} N_{e}$. The median cosmic-ray energy, $E_{C R}$, and the median $\gamma$-ray energy, $E_{\gamma}$, are given in the fifth and sixth columns, respectively, in units of TeV. The quantities $N_{90}$ and $\varepsilon_{\gamma}$ are defined in the text. $I_{\gamma} / I_{C R}$ is the $90 \%$ C.L. upper limit on the integral $\gamma$-ray fraction, and $I_{\gamma}$ is the $90 \%$ C.L. upper limit on the integral $\gamma$-ray flux, in units of photons $\mathrm{cm}^{-2} \mathrm{~s}^{-1} s r^{-1}$.

\begin{tabular}{lccccccc}
\hline \hline $\log _{10} N_{e}$ & $N_{\text {tot }}$ & $N_{90}$ & $\varepsilon_{\gamma}$ & $E_{C R}$ & $E_{\gamma}$ & $I_{\gamma} / I_{C R}$ & $I_{\gamma}$ \\
\hline$>4$ & $1.02 \times 10^{8}$ & 11653.5 & 0.33 & 333 & 248 & $<2.1 \times 10^{-4}$ & $<4.02 \times 10^{-13}$ \\
$>4.5$ & $2.19 \times 10^{7}$ & 583.3 & 0.27 & 783 & 605 & $<6.3 \times 10^{-5}$ & $<2.67 \times 10^{-14}$ \\
$>5$ & $3.92 \times 10^{6}$ & 19.0 & 0.18 & 1994 & 1502 & $<1.7 \times 10^{-5}$ & $<1.46 \times 10^{-15}$ \\
$>5.5$ & $5.76 \times 10^{5}$ & 2.3 & 0.20 & 5247 & 3673 & $<1.1 \times 10^{-5}$ & $<1.73 \times 10^{-16}$ \\
$>6$ & $6.75 \times 10^{4}$ & 2.3 & 0.44 & 14618 & 8603 & $<3.1 \times 10^{-5}$ & $<8.77 \times 10^{-17}$ \\
$>6.5$ & $6.66 \times 10^{3}$ & 2.3 & 0.44 & 44952 & 18610 & $<8.1 \times 10^{-5}$ & $<1.47 \times 10^{-17}$ \\
\hline
\end{tabular}

\section{Conclusion}

Using the data sets measured by the KASCADE experiment between 1996 October and 2010 May, whose muon content has been measured by a large muon detector, we place stringent upper limits $\left(10^{-5}-10^{-4}\right)$ on the $\gamma$-ray fraction of the cosmic rays at energies between $\sim 2.5 \times 10^{14} \mathrm{eV}$ and $\sim 1.9 \times 10^{16} \mathrm{eV}$. The upper limit of the fraction of $\gamma$-rays at $1.5 \times 10^{15} \mathrm{eV}$ is $1.7 \times 10^{-5}$, while $1.1 \times 10^{-5}$ at $3.7 \times 10^{15} \mathrm{eV}$. These are the lowest upper limits in the world up to now, which may set some constraints on the fundamental astrophysical model, such as the distance of sources for the IceCube neutrino excess model [ㄹ3].

\section{References}

[1] Berezinsky V.S. et al., Astropart. Phys. 1 (1993) 281

[2] Halzen A.F. et al., Phys. ReV. D 41 (1990) 342

[3] Sigl G., Schramm D.N., and Bhattacharjee P., Astropart. Phys. 2 (1994) 401

[4] Antoni, T., et al. 2003, Nucl. Instrum. Methods Phys. Res. A, 513, 490

[5] Greisen, K. 1956, in Progress in cosmic ray Physics 3, ed. J. G. Wilson (Amsterdam: North-Holland)

[6] Kamata, K., \& Nishimura, J. 1958, Prog. Theor. Phys. Suppl., 6, 93

[7] Antoni, T., et al. 2001, Astropart. Phys., 14, 245

[8] Heck D, Knapp J, Capdevielle J N, Schatz G, Thouw T, 1998, Report FZKA 6019

[9] Antoni, T., et al., Astroparticle Physics 24 (2005) 11́C25

[10] Glasstetter, R., cosmic ray Energy And Mass Estimator, http://www-ik.fzk.de/ ralph/CREAM.php.

[11] Antoni, T., et al., 2003, Astropart. Phys., 19, 703

[12] O. Helene, Nucl. Instr. Meth. 212 (1983) 319

[13] M. C. Chantell et al. , Phys. Rev. Lett. 79, 1805 (1997)

[14] C. Amsler et al. (Particle Data Group), Physics Letters B667, 1, (2008) 
[15] M. Aglietta et al. , Astropart. Phys. 6, 71 (1996).

[16] A. Karle et al. , Phys. Lett. B 347, 161 (1995)

[17] , F. A. Aharonian et al. , Astropart. Phys. 17, 459 (2002)

[18] J. Matthews et al. Astrophys. J. 375, 202 (1991).

[19] M. Minamino et al. , Pro. of the 31st Int. Cosmic Ray Conf., Lodz, Poland (2009).

[20] Yu.A. Fomina, N.N. Kalmykova, G.V. Kulikova, V.P. Sulakova and S.V. Troitskyb, arXiv:1307.4988v1

[21] D. Kang et al. KASCADE-Grande Collaboration, Proc. 34th Int. Cosmic Ray Conf., The Hague, The Netherlands (2015), ID785 of these proceedings.

[22] G. Schatz et al., KASCADE Collaboration, Proc. 28th Int. Cosmic Ray Conf., Tsukuba, Japan (2003).

[23] Ahlers, M., \& Murase, K. 2014, PRD, 90, 023010 